\title{
Design and Performance of a Block-Type Osmometer
}

\author{
Donald McIntyre, G. C. Doderer, ${ }^{1}$ and James H. O'Mara
}

\begin{abstract}
A block-type osmometer has been designed and constructed utilizing several new features which enhance the precision of the osmotic-pressure measurement and allow simpler manipulative techniques. The details of the construction as well as a discussion of the design and performance of this osmometer are included in the paper.
\end{abstract}

\section{Introduction}

An improved block-type osmometer has been designed and constructed that is capable of making precise measurements of the osmotic pressures of polymer solutions. This osmometer was designed so as to make the measurements as free as possible of determinate errors without necessarily complicating the laboratory manipulative work. It incorporates many of the desirable features obtainable with other osmometer designs. In undertaking this work it was realized that the semipermeable membrane is the ultimate controlling factor in any osmotic-pressure measurement, but it was also known that a good osmometer is essential in order to obtain a reliable evaluation of membranes. This new osmometer was also constructed so that comparative data might be obtained to evaluate the performance of commercially available osmometers.

\section{Construction of Block Osmometer}

An osmometer must provide two compartments which are separated by a semipermeable membrane. When a solvent is placed in one compartment and a solution containing an impermeable solute is placed in the other, equilibrium between these two liquid phases can be established by applying a pressure to the solution which has a lower solvent chemical potential. This pressure is called the osmotic pressure, and can be created by the transfer of solvent through the membrane to cause a height difference of the solutions in the capillaries connected to the compartments, or by the application of external pressure through a manometer. The present osmometer can use either method of pressure measurement. In figure 1, a portion of the machinist's drawing for the osmometer is reproduced, and in figure 2, the assembled and open views of the osmometer are shown. The solution and solvent are introduced into each half of the osmometer through the standpipe, located at $\mathrm{C}$, which is subsequently closed by the needle valve, located at D. The osmotic pressure is then measured as the height difference in the two capillaries, connected to the blocks at E. The wafflelike blocks that clamp the membrane were made of $440 \mathrm{C}$ hardened stainless steel. The adapter, nut, needle, and standpipe were made of 303 stainless steel. The sleeve that joins the uniform-bore capillary through a graded seal to the adapter was made of Kovar. The needle

${ }_{1}^{1}$ Present address: General Electric Company, Louisville, Ky.

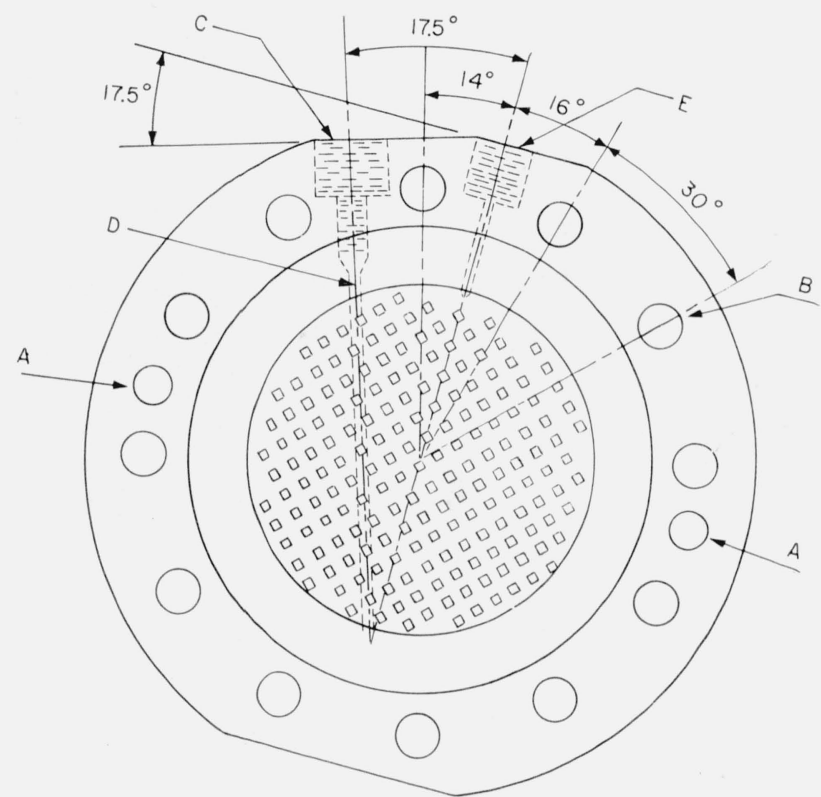

Figure 1. Drawing of osmometer.

A, Guide pins; B, bolt holes; C, standpipe connection; D, needle valve seat; $\mathrm{E}$, capillary connection.

valves were made of a soft stainless-steel needle bearing against the hard stainless-steel block.

Stainless steel was used as the basic material in order to make the osmometer useful for aqueous solutions, and also to obtain the necessary hardness for polishing the faces of the osmometer. Initial plans were to use a more corrosion-resistant but hardenable stainless steel in the 400 series, but the desired stainless steel was not available at the time. As a consequence the corrosion resistance is not as great as had been hoped for, but proved to be adequate after the whole surface was well finished and free of machining marks.

Two osmometers were constructed. The first osmometer was hardened in an "inert" atmosphere at the National Bureau of Standards. The second osmometer was hardened at the United States Naval Gun Factory in a salt bath with an additional second tempering step to avoid possible stress from hardening. The hardness of both was about 52 to 54 Rockwell C.

After the hardening operations, the first osmometer was ground by using a magnetic chuck, and the second was ground with a regular chuck in order to avoid the induced magnetization which makes the Internal parts more difficult to clean. However, the 


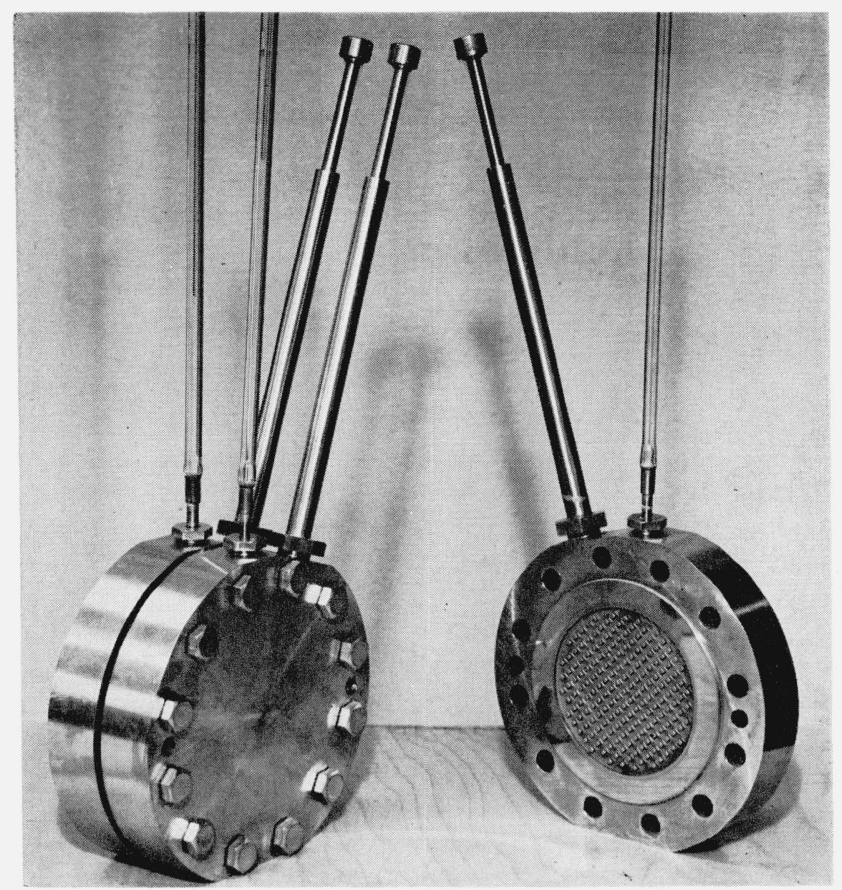

Figure 2. Fully assembled osmometer (left) and half of an osmometer (right).

magnetic chucking enabled a better polished surface and seems preferable for the best final results. The surfaces were finally ground planar within approximately one-half of a fringe of white light over the whole area. An unexpected problem arose during the grinding of the flat surfaces. A concave surface was produced in the center of the osmometer because the small pillars decreased the resistance to the grinder and also because the heat treating tended to transform the outer layer into a harder austenitic phase than the center.

\section{General Performance}

All performance tests were conducted with gel "never-dried" cellophane No. 600 membranes. These membranes were very impermeable to solvent and had "zero-point" corrections in this osmometer of always less than $0.05 \mathrm{~mm}$, and usually $0.00 \pm 0.02$ $\mathrm{mm}$. At first the Kovar metal sleeve that was immersed in the water bath began to corrode badly by pitting. After a few unsuccessful remedies, the Kovar was nickel plated and then coated with resin at the glass seal, where the plating was least effective. This proved to be successful. Perhaps a better method of sealing the capillary to the osmometer would have been the one used by Krigbaum and Flory $[1],{ }^{2}$ who formed a foot on the capillary itself and eliminated a glass-to-metal seal.

Initially, the first osmometer was tested without removing the scale, which was caused by oxidation during the hardening process, from the outside of the osmometer. However, bubbles immediately began to form within the osmometer after its immer-

2 Figures in brackets indicate the literature references at the end of this paper. sion in water. This condition was accompanied by corrosion which never did cease. Later the scale was removed and the osmometer reassembled, but it was two weeks before the gas from the corrosion finally diffused out of the blocks. The second osmometer, which had no oxidized scale, never showed these effects.

The filling and emptying of the osmometer was easily done with a long hypodermic needle with a blunt end. This procedure avoided any bubbles. Each side of the osmometer required about $7 \mathrm{ml}$ to fill the block, although more was required to fill the standpipe and capillary. About $13 \mathrm{ml}$ was used for each side, and the excess liquid was removed from each standpipe as soon as the measurement was underway. The needle valves were tightened with a torque wrench to 18 to $20 \mathrm{lb}$-in. The osmometer did not need to be removed from the bath during the filling and emptying operations, so that osmoticpressure measurements at nonambient temperatures were easily made without any severe dilatometric problems. The temperature variation of the bath was kept to $\pm 0.002^{\circ} \mathrm{C}$ in order to eliminate the thermometric effect in the $0.5 \mathrm{~mm}$ - and $1.0 \mathrm{~mm}$ diameter capillaries used with the osmometer.

Measurements were based upon the difference in height between the vertical solution and solvent capillaries using cathetometers that gave an accuracy of $\pm 0.02 \mathrm{~mm}$. During the measurements on solvent permeabilities it was found that the sum of the heights of the liquids in the two capillaries of the block osmometer immersed in the water bath slowly increased with time, at about the rate of 0.2 to 0.4 $\mathrm{mm} / \mathrm{hr}$. This was so slow that the membrane dissymmetry (or even osmotic pressures) did not change with time once equilibrium had been reached. It was found that the liquid levels decreased extremely fast $(6.5 \mathrm{~mm} / \mathrm{hr})$ when the osmometer was imbedded in a thermostatted box filled with cotton. These observations pointed to the fact that there was permeation of the solvent through the edges of the membrane. When the tests in the cotton-filled box were modified so as to have cyclohexane vapor in the area surrounding the membrane, the leakage rate dropped to practically zero. Nevertheless, after several weeks of operation with the osmometer directly immersed in water without any additional sealing of the membrane edge, the osmometer was opened for an examination of the membrane; it showed no traces of water permeation. The membrane apparently is securely protected against bath fluid penetration by the tight seal and by the additional head supplied by the solvent in the capillaries.

In order to prevent permeation through the edges of the membrane, a special gasket containing a metal foil encircling the membrane around the outer edge was used. The results obtained were similar to those obtained when polyvinyl alcohol was coated on the outer edge of the optical flats after the osmometer was assembled. This was tested when the osmometer was inserted into a xylene bath as well as in a silicone oil bath, with identical results. All subsequent measurements have been conducted in a silicone oil thermostat. With these precautions 
the decrease in the sum of the capillary heights is $0.2 \mathrm{~mm} / \mathrm{hr}$ when $0.5-\mathrm{mm}$ capillaries are used with cyclohexane. Undoubtedly some of this decrease is due to evaporation of the cyclohexane. However, it does seem necessary to have a gasket or sealing of some sort on any block osmometer to prevent slow permeations through the edge of the membrane.

\section{Osmotic-Pressure Measurements}

Osmotic-pressure measurements were made with two different types of osmometer. The special block osmometer described earlier was tested and then used to evaluate the operation of a commonly used osmometer of different construction [2]. The Stabin osmometers performed well when they were assembled with the same type of membrane (gel cellophane) as that used for the NBS reference block osmometer. The membrane dissymmetries after 2 or 3 days were the same as those of the NBS osmometer, that is, less than $0.05 \mathrm{~mm}$ of toluene or cvclohexane. The Stabin osmometers were easily assembled and worked well from the beginning. A mercury seal was used around the positioning rod.

Figure 3 shows the results of osmotic-pressure measurements on a polystyrene fraction in toluene at $30^{\circ} \mathrm{C}$ with two Stabin osmometers and the new block osmometer. In this figure, $\mathrm{h}$ is the difference in heights of toluene in the two capillaries in centimeters while $C$ is the concentration in grams per deciliter. Figure 4 shows the same fraction of polystyrene in cyclohexane determined at $40^{\circ} \mathrm{C}$ with the two different types of osmometers. Figure 5 shows the results of the osmotic-pressure measurements on a fraction of polystyrene in cyclohexane at different temperatures using different osmometers.

Table 1 indicates the speed with which solvent permeates the membrane in the respective osmometers. The constant, $k$, is determined from the slope of the curve represented in eq (1), $h$ being the difference in column levels in centimeters at time, $t$, in seconds.

$$
\ln h=l \cdot t
$$

Both types of osmometers achieved equilibrium osmotic pressures in cyclohexane within 5 to 7 days.

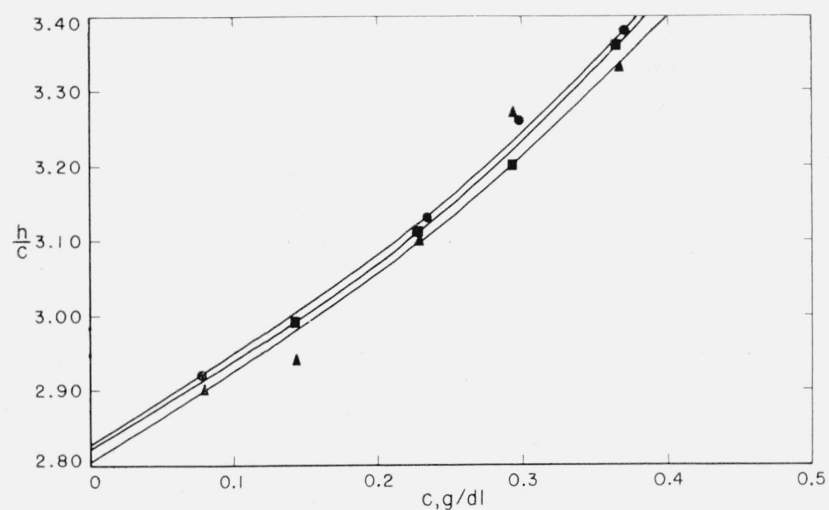

Figure 3. Osmotic-pressure measurements on a polystyrene fraction in toluene at $30^{\circ} \mathrm{C}$ using the different types of osmometers.

A, NBS block; Stabin; Stabin.

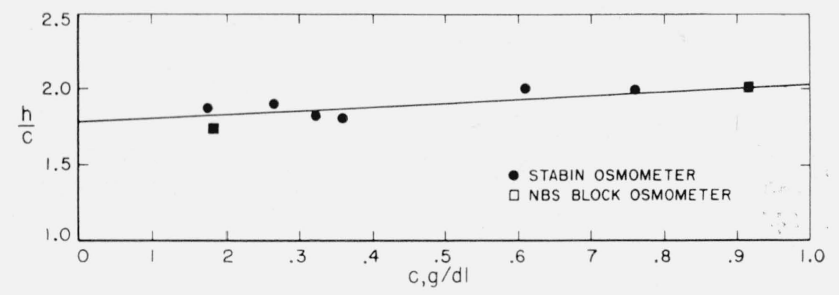

Figure 4. Osmotic-pressure measurements on a polystyrene fraction in cyclohexane at $40^{\circ} \mathrm{C}$ using the different types of osmometers.

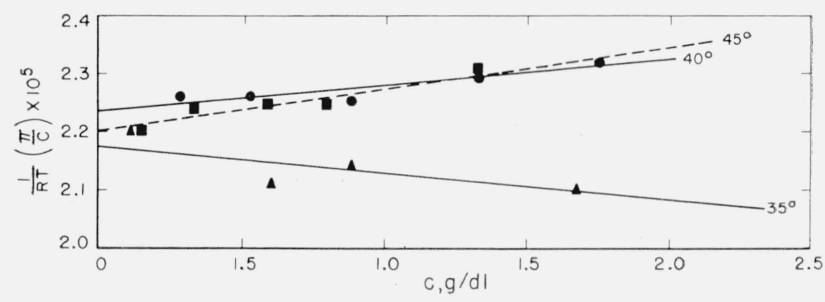

Figure 5. Osmotic-pressure measurements on a polystyrene fraction in cyclohexane at different temperatures using different osmometers.

$35^{\circ}$, Stabin osmometer; $40^{\circ}$, Stabin osmometer; $45^{\circ}$, NBS osmometer.

although the NBS block osmometer approached 95 percent of the equilibrium value within 1 or 2 days and the Stabin osmometer in 2 to 3 days. The solu tion concentrations for each osmometer were de termined initially by weight and also by differential refractive index measurements in an interferometer. After a run, both the solvent and solution in the block osmometer were checked interterometrically for changes or errors. The refractive indices in the NBS block osmometer checked within $5.6 \times 10^{-6}$ units, which for cyclohexane-polystyrene solutions would correspond to a change in concentration of 0.0033 percent if permeation of the polystyrene molecules alone accounted for the refractive index change. However, the limiting factor in such analyses is not the interferometry but rather the small changes that occur in solvents upon being handled or being in contact with metal. These changes are due either to absorption of contaminants or actual chemical reactions which alter the solvent.

TABLE 1. Permeability constants for cyclohexane

\begin{tabular}{|c|c|}
\hline \multicolumn{1}{|c|}{ Osmometer } & $k$ \\
\hline Stabin 1 & $\mathrm{cm} / \mathrm{sec}$ \\
Stabin 2 & $-3.3 \times 10^{-5}$ \\
NBS block 1 $1 \ldots .6 \times 10^{-5}$ \\
\end{tabular}

\section{Discussion}

\subsection{Design}

Osmometer design can basically be resolved into (1) the manner of supporting the semipermeable membrane, (2) the critical dimensions of the cell, which determine the membrane surface to cel! volume ratio, (3) the mechanism of filling and emptying the osmometer, and (4) the method of measuring the osmotic pressure. Before discussing 
the design features in the NBS block osmometer it would seem necessary to point out that the design considerations were based upon the empirical knowledge that the final limit of error resides in the "zeropoint correction", or "membrane dissymmetry". This factor, even with a well-conditioned membrane, may be unknown to as much as $\pm 0.05 \mathrm{~mm}$ height difference of an organic solvent like toluene or cyclohexane. In view of this uncertainty, the measurement of the osmotic pressure need only involve a resolution of $\pm 0.02 \mathrm{~mm}$ of solvent.

The membrane is obviously best supported when both sides are firmly held in place to avoid "ballooning" or other relaxation effects. Meyer [3], Fuoss and Mead [4], Flory [5], Carter and Record [6], and Trementozzi [7] have successfully used milled metal blocks to support the membranes. Various types of milled designs have been used. In spite of a slight loss in membrane rigidity the present investigators undertook the more costly operation of milling small pillars rather than circular grooves in order to gain the definite advantage of easier bubblefree filling. Demonstrations with a completed block, i. e., half an osmometer, sealed with a glass plate have visually shown the complete avoidance of bubbles in the filling.

Since the membranes are supported on optical flats it seemed that this tight seal of the membrane would make additional gaskets on the outer periphery unnecessary. Other workers [8] have stated the need for such a gasket, and the present investigation has also demonstrated the need. even though other osmometers have been reported to operate successfully without the use of an additional seal $[4,5,7]$.

This type of membrane support has proved effective in avoiding drastic relaxation effects in the membrane. The low membrane dissymmetries and the quick approach of the zero point to a constant value have been encouraging proof of this point. In addition it appears that the membranes currently being used are highly relaxed before they are introduced into the osmometer since the low zero-point correction is quickly established after 2 or 3 davs and several solvent rinses. The development of the more rigid inorganic or glass membranes would, of course, eliminate the need of such precision finishing of the blocks, but after many years these membranes are still not yet widely used nor have they been critically tested.

The surface area of the membrane that is directly exposed to solution is approximately $41 \mathrm{~cm}^{2}$ and the volume of solution is approximately $7 \mathrm{ml}$, so that the surface/volume ratio is about comparable to that of other block osmometers. The effect or role of adsorption has not been studied enough to allow any more seientific choice of surface/volume ratios other than not making them too large.

The ease of filling and emptying, as well as the ability to make an accurate concentration anaylsis, has been a real justification for the attempt to improve the block osmometer. The NBS model has shown that high quality workmanship can produce an instrument that has a low leakage and volatili- zation rate with organic liquids, even those having boiling points as low as that of cyclohexane. Devices built into the capillaries to avoid solvent evaporation have not proved necessary, even in work with cyclohexane at $45^{\circ} \mathrm{C}$.

The block-type osmometer has the added advantage that the pressure necessary for equalizing the solvent and solution activities may be easily applied externally as well as through the difference of the heights in the capillaries. It is planned later to extend the range of osmotic pressures by adding external pressure to the capillaries.

The heavy metal blocks of the osmometer are very useful in keeping a constant-temperature profile on both sides of the membrane throughout the region where the osmotic pressure is being created. A small temperature differential can mask the difference in activity between solvent and solution, which is precisely the quantity that one is trying to equalize by a pressure increase. In the nonblock osmometers the lagging due to the interposed solvent partially compensates for this lack of metal, but certainly the block osmometer is to be desired for critical work.

\subsection{Comparative Measurements}

As the data in figures 3,4 , and 5 indicate, osmoticpressure measurements using polymer fractions with the two osmometers are indistinguishable within experimental error. This makes routine osmoticpressure measurements valid with either osmometer. However, for unfractionated materials a block osmometer becomes extremely valuable because it facilitates the detection of solute permeation.

As an instrument for testing membranes and their permeability to solvent and solute species, the block osmometer can be extremely valuable. In addition, this osmometer can function easily as a null osmometer for dynamic measurements at any temperature compatible with the membrane.

The authors express their thanks to W. Gnueg of the NBS machine shop and E. Webb and his coworkers in the NBS optical shop for their cooperation and expert construction work. The authors also express their appreciation to L. H. Fawcett and his group at the United States Naval Gun Factory for their cooperation in hardening the steel for the osmometers.

\section{References}

[1] W. R. Krigbaum and P. J. Flory, J. Am. Chem. Soc. 75 , 1775 (1953).

[2] J. V. Stabin and E. H. Immergut, J. Polymer Sci. 14, 209 (1954).

[3] K. H. Meyer, E. Wolff, C. G. Boissonnas, Helv. Chim. Acta. 23, 430 (1940).

[4] R. M. Fuoss and D. J. Mead, J. Phys. Chem. 47, 59 (1943).

[5] P. J. Flory, J. Am. Chem. Soc. 65, 372 (1943).

[6] S. R. Carter and B. R. Record, J. Chem. Soc. 1939, 660.

[7] Q. A. Trementozzi, Monsanto Chemical Co., Springfield, Mass. (private communication).

[8] A. F. Sirianni, L. M. Wise, and R. L. MeIntosh, Can. J. Research [B] 25, 301 (1947).

Washington, October 28, 1958. 